Pacific Journal of Mathematics

FIBER INTEGRATION IN SMOOTH BUNDLES 


\title{
FIBER INTEGRATION IN SMOOTH BUNDLES
}

\author{
J. W. AUER
}

The purpose of this paper is to comment on the operations of flber integration, or integration over the fiber, which arise in the study of the cohomology of bundles.

Let $\xi=\left(E, \Pi_{E}, B, F, G\right)$ be a smooth $\left(C^{\infty}\right)$ bundle, where as usual $E$ is the total space, $B$ the base, $F$ the (connected) fiber, $G$ the group, and $\Pi_{E}: E \rightarrow B$ the projection. Assume $H^{k}(F)$ to be finite dimensional for all $k$. A form $\omega$ on the total space is said to have fiber-compact support if, and only if, for all $x \in B$, there is a neighbourhood $U_{x}$ of $x$, a trivialization $\phi: U_{x} \times F \cong \pi_{E}^{-1}\left(U_{x}\right)$, and a compact set $K \subset F$ such that (support $\left.\phi^{*} \omega\right) \cap\left(U_{x} \times F\right) \subset U_{x} \times K$. Denote these $k$-forms by $A_{F}^{k}(E)$, and their de Rham cohomology by $H_{F}^{k}(E)$. When $F$ is compact $A_{F}^{k}(E)=A^{k}(E)$, the algebra of all $k$-forms on $E$; if $B$ is compact, $A_{F}(E)$ is the algebra $A_{c}(E)$ of forms on $E$ with compact support. Now integration over fiber has been defined by various authors as a linear map

$$
\Psi: H^{k}(E) \longrightarrow H^{k-m}\left(B ; H^{m}(F)\right), k \geqq m,
$$

where $m$ is the dimension of $F$. These definitions are essentially algebraic in nature; for example $\Psi$ has been defined by a spectral sequence. Using this idea when $\xi$ is orientable, a linear map

$$
\Psi_{1}: H_{F}^{k}(E) \longrightarrow H^{k-m}(B)
$$

is defined, and called algebraic fiber integration on account of the origin of the definition.

On the other hand, when $\xi$ is orientable, there is the geometrically defined linear map $f_{F}: A_{F}^{k}(E) \rightarrow A^{k-m}(B)$ given roughly speaking by

$$
\left(f_{F} \omega\right)(x)=\int_{F_{x}} \omega / F_{x},
$$

where $\omega \in A_{F}^{k}(E), x \in B$, and $F_{x}$ denotes the fiber of $\xi$ over $x$. The induced map $\Psi_{2}$ of cohomology is called geometric fiber integration:

$\Psi_{2}: H_{F}^{k}(E) \rightarrow H^{k-m}(B)$. The main purpose of the paper is to show

THEOREM. $\Psi_{1}=\Psi_{2}$.

1. The spectral sequence in $A_{F}(E)$. 
(a) In this section we obtain results analogous to a theorem of Borel [6], which we use to obtain an expression for $\Psi_{1}$.

The action of $G$ on $F$ induces an action on $H^{k}(F)$ and on $H_{c}^{k}(F)$, $k=0,1,2, \cdots, m$, where $H_{c}^{k}(F)$ denotes the de Rham cohomology of forms on $F$ with compact support. Denote by $h^{k}$ the total space of the bundle over $B$ with fiber $H_{c}^{k}(F)$; because this bundle has a discrete group, the exterior derivative $\delta_{B}^{p, q}$ in $A_{B}^{p}\left(h^{q}\right)$ (the $p$-forms on $B$ witn coefficients in $h^{q}$ ) is a differential operator. Denote $\operatorname{Ker} \delta_{B}^{p, q} / \operatorname{Im} \delta_{B}^{p-1, q}$ by $H^{p}\left(B ; h^{q}\right)$.

(b) We filter $A_{F}^{k}(E)$ following Hattori [5] and Borel [6]: when $\left\{x_{i}\right\},\left\{y_{j}\right\}$ are coordinates on neighbourhoods in $B$ and $F$ respectively, $i=1,2, \cdots, n-m, j=1,2, \cdots, m$, where $n=\operatorname{dim} E$, we use the same symbols to denote coordinates induced on sufficiently small open sets in $E$; then according to Hattori and Borel, $I^{p}$ consists of those forms which involve at least $p$ base differentials $d x_{i}$.

It will be convenient to define this filtration by a bigradation of $A_{F}(E)$. For this purpose, it is necessary to assume that a fixed connection ([7], p. 63) has been prescribed in $\xi$. Of course, by the remarks above, the filtration will be independent of the connection. If $X$ is a $C^{\infty}$ vector field on $E$, then by definition its horizontal and vertical parts, $H X$ and $V X$ respectively, induced by the connection, are again $C^{\infty}$ vector fields. Whenever $\left\{x_{i}\right\},\left\{y_{j}\right\}$ are coordinates on an open set $W \subset E$, as described above, we denote by $d y_{j}^{v}, j=1,2, \cdots$, $m$, the 1 -forms on $W$ defined by $d y_{j}^{v}(x)=d y_{j}(V X)$, where $X$ is a vector field on $W$. Then $\left\{d x_{i}\right\} \cup\left\{d y_{j}^{v}\right\}$ generate $A^{1}(W)$ over $C^{\infty}(W)$.

Definition 1. $C^{p, q} \equiv C^{p, q}(E)=\left\{\omega \in A_{F}^{p+q}(E) \mid i\left(X_{1}\right) \cdots i\left(X_{p+1}\right) \omega=\right.$ $i\left(Y_{1}\right) \cdots i\left(Y_{q+1}\right) \omega=0$ for all horizontal vector fields $X_{i}$ and vertical vector fields $Y_{j}$ on $E$ \}.

Here, as usual, $i(Z)$ is the substitution operator with respect to the vector field $Z$ on $E$ :

$$
i(Z) \omega\left(Z_{1}, \cdots, Z_{p+q-1}\right)=\omega\left(Z, Z_{1}, \cdots, Z_{p+q-1}\right)
$$

when $Z_{1}, \cdots, Z_{p+q-1}$ are vector fields on $E, \omega \in A^{p+q}(E)$. Thus $i(Z)$ : $A_{F}^{p+q}(E) \rightarrow A_{F}^{p+q-1}(E)$, because $A_{F}(E)$ is clearly stable under $i(Z)$.

Now we let Greek letters $\alpha, \beta$, etc., represent sequences of positive integers of the form $\left(i_{1}, \cdots, i_{p}\right)$, for some positive integer $p$, with $i_{1}<i_{2}<\cdots<i_{p}$. Then $d x_{\alpha}$ will denote $d x_{i_{1}} \wedge \cdots \wedge d x_{i_{p}}$, and so on. We put $|\alpha|=p$ when $\alpha=\left(i_{1}, \cdots, i_{p}\right)$. Then if $W$ is a coordinate neighbourhood in $E, \omega \in A^{k}(E)$, we may write $\omega / W$ as

$$
\omega / W=\sum_{p+q=k} \sum_{\substack{|\alpha|=p \\|\beta|=q}} \omega^{\alpha \beta} d x_{\alpha} \wedge d y_{\beta}^{v}
$$


where $\omega^{\alpha \beta} \in C^{\infty}(W)$, the $C^{\infty}$-functions on $W$. Consequently any $\omega \in C^{p, q}(W)$ may be written

$$
\omega=\sum_{\substack{|\alpha|=p \\|\beta|=q}} \omega^{\alpha \beta} d x_{\alpha} \wedge d y_{\beta}
$$

with $\omega^{\alpha \beta} \in C^{\infty}(W)$.

\section{DEFINITION 2.}

$$
I^{p, q}=\bigoplus_{r \geqq p} C^{r, p+q-r}, \quad I^{p}=\bigoplus_{q \geq 0} I^{p, q} .
$$

It is easily seen that

Proposition 1. $\left\{I^{p}\right\}_{p=0}^{\infty}$ is a decreasing filtration of $A_{F}(E)$. It is the filtration associated with the gradation $\left\{C^{p}\right\}_{p=0}^{\infty}$ of $A_{F}(E)$, where $C^{p}=\bigoplus_{q \geq 0} C^{p, q}$, because $I^{p}=\bigoplus_{r \geqq p} C^{r}$.

Note in particular that $I^{0}=A_{F}(E)$, and $I^{p}=\{0\}$ for $p>\operatorname{dim} B=$ $n-m$.

REMARK. If the above definitions are carried out for $A(E)$, one obtains the filtrations of Hattori [5] and Borel [6]. In particular, the filtration is independent of the choice of connection in $\xi$.

(c) Denote by $\left\{E_{r}^{p, q}\right\}$ the spectral sequence defined by the filtration $\left\{I^{p, q}\right\}$ of $A_{F}(E)$ defined above (see $\S 2$ ). Then we will next show

\section{Proposition 2.}

$$
E_{2}^{p, q} \cong H^{p}\left(B ; h^{q}\right), \quad p, q \geqq 0 .
$$

Let $\mathscr{X}_{B}{ }^{P}$ be the sheaf of germs of $p$-forms on $B$, and $\mathscr{C}_{B}{ }^{\infty}$ the sheaf of germs of $C^{\infty}$-functions on $B$.

Definition 3. The sheaf $\mathscr{F}^{q}$ of fiber-compact $q$-forms along the fiber of $\xi$.

We first define the presheaf $\mathscr{F}^{q}$ on $B$ by $\mathscr{F}^{q}(U)=C^{0, q}\left(\Pi_{E}^{-1}(U)\right)$, when $U$ is open in $B$. Then $\mathscr{F}^{q}$ is the sheaf induced by $\mathscr{F}^{q}$.

Lemma 1. $E_{0}^{p, q} \cong \Gamma\left(\mathscr{A}_{B}^{P} \otimes \mathscr{F}^{q}\right)(\Gamma(\mathscr{S})$ denotes the module of sections of any sheaf $\mathscr{S}$, and all tensor products are over the sheaf $\left.\mathscr{C}_{B}^{\infty}\right)$.

Proof. From the definitions we have that 


$$
E_{0}^{p, q}=C^{p, q}
$$

DeFinition 4.

$$
\Omega: \Gamma\left(\mathscr{A}_{B}{ }^{p} \otimes \mathscr{F}^{q}\right) \longrightarrow C^{p, q} .
$$

Let $s \in \Gamma\left(\mathscr{A}_{B}^{P} \otimes \mathscr{F}^{q}\right)$; then if $U \subset B$ is sufficiently small, we may write $s$ locally as a sum of terms of the form $\omega$, where

$$
\omega=\left(\sum_{|\alpha|=p} \omega_{1}^{\alpha} d x_{\alpha}\right) \otimes\left(\sum_{|\beta|=q} \omega_{2}^{\beta} d y_{\beta}^{v}\right)
$$

with $\omega_{1}^{\alpha} \in C^{\infty}(U), \omega_{2}^{\beta} \in C^{\infty}\left(\Pi_{E}^{-1}(U)\right)$. Put

$$
\Omega(\omega)=\sum_{\substack{|\alpha|=p \\ \beta \mid=q}}\left(\omega_{1}^{\alpha} \Pi_{E}\right) \omega_{2}^{\beta} d x_{\alpha} \wedge d y_{\beta}^{v}
$$

and extend linearly to define $\Omega(s)$.

Lemma 2. $\Omega$ is well-defined, independent of the choice of coordinates.

Proof. This follows from the fact that if $\left\{\bar{x}_{i}\right\},\left\{\bar{y}_{j}\right\}$ are coordinates defined on an open set in $E$ overlapping the domain of definition of the coordinates $\left\{x_{i}\right\},\left\{y_{j}\right\}$, then

$$
d \bar{x}_{\alpha}=\sum_{|\mu|=p} \frac{\partial \bar{x}_{\alpha}}{\partial x_{\mu}} d x_{\mu}
$$

and

$$
d \bar{y}_{\beta}^{v}=\sum_{|\alpha|=q} \frac{\partial \bar{y}_{\beta}}{\partial y_{\alpha}} d y_{\alpha}^{v}
$$

where $\partial \bar{x}_{\alpha} / \partial x_{\mu}$ represents the $p \times p$ sub-matrix with rows $\alpha$, columns $\mu$ of the $(n-m) \times(n-m)$ matrix with entries $\partial \bar{x}_{i} / \partial x_{j}$, and analogously for $\partial \bar{y}_{\beta} / \partial y_{\alpha}$. Note that equation (4) is not the equation of transformation for the $d y_{\beta}$ 's; the latter equation also involves linear combinations of the $d x_{\alpha}$ 's. Since $\Omega$ is easily seen to be an isomorphism, this completes the proof of Lemma 1.

DeFINITION 5. The homomorphism

$$
\delta_{F}^{p, q}: \Gamma\left(\mathscr{A}_{B}^{P} \otimes \mathscr{F}^{q}\right) \longrightarrow \Gamma\left(\mathscr{A}_{B}^{P} \otimes \mathscr{F}^{q+1}\right) .
$$

We first define the presheaf homomorphism $\underline{\delta}_{F}^{q}: \mathscr{F}^{q} \rightarrow \mathscr{F}^{q+1}$. Let $U \subset B$ be open, $\phi \in \mathscr{F}^{q}(U)=C^{0, q}\left(\Pi_{E}^{-1}(U)\right)$, and $Y_{1}, \cdots, Y_{q+1}$ be vertical vector fields on $\Pi_{E}^{-1}(U)$. Then 


$$
\begin{aligned}
& \delta_{F}^{q}(U)(\phi)\left(Y_{1}, \cdots, Y_{q+1}\right) \\
= & \frac{1}{q+1} \sum_{i=1}^{q+1}(-1)^{i-1} Y_{i}\left(\phi\left(Y_{1}, \cdots, \hat{Y}_{i}, \cdots, Y_{q+1}\right)\right. \\
& +\frac{1}{q+1} \sum_{i<j}(-1)^{i+j} \phi\left(\left[Y_{i}, Y_{j}\right], Y_{1}, \cdots, \hat{Y}_{i}, \cdots \hat{Y}_{j}, \cdots, Y_{q+1}\right)
\end{aligned}
$$

where the symbol $\hat{Y}_{i}$ indicates that $Y_{i}$ is to be omitted. In terms of coordinates, if $\phi=\sum_{|\beta|=q} \omega^{\beta} d y_{\beta}^{v}$, with $\omega^{\beta} \in C^{\infty}\left(\Pi_{E}^{-1}(U)\right)$, then

$$
\underline{\delta}_{F}^{q}(U)(\phi)=\sum_{|\beta|=q} \sum_{k} \frac{\partial \omega^{\beta}}{\partial y_{k}} d y_{k}^{v} \wedge d y_{\beta}^{v}
$$

Now $\delta_{F}^{q}$ is the sheaf homomorphism induced by $\underline{\delta}_{F}^{q}$, and $\delta_{F}^{p, q}$ is the homomorphism of modules of sections induced by $(-1)^{p} 1_{\mathscr{S}_{B}^{p}} \otimes \delta_{F}^{q}$.

Let $\Delta_{0}^{p, q}: E_{0}^{p, q} \rightarrow E_{0}^{p, q+1}$ be the map induced by the exterior derivative on $E$; that is, $\Delta_{0}^{p, q}$ is defined by the diagram below, where $\rho_{0}^{p, q}$ is the canonical projection:

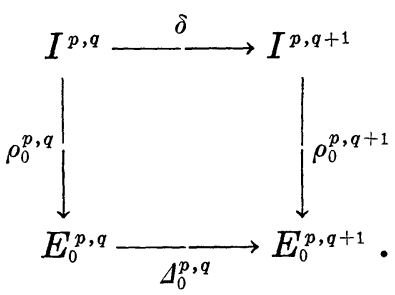

Now in view of $E_{0}^{p, q}=C^{p, q}, \Delta_{0}^{p, q}$ is exactly the differential operator induced in $C^{p}$ by the exterior derivative on $E$ :

$$
\rho^{p, q+1} \delta=\Delta_{0}^{p, q}: C^{p, q} \longrightarrow C^{p, q+1},
$$

where $\rho^{p, q+1}: A_{F}^{p+q+1}(E) \rightarrow C^{p, q+1}$ is the canonical projection induced by $A_{F}(E)=\bigoplus_{p, q} C^{p, q}$.

LEMma 3. The diagram below is commutative:

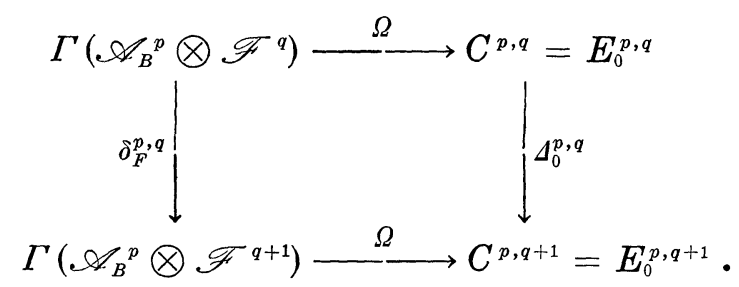

Proof. Any $s \in \Gamma\left(\mathscr{A}_{B}{ }^{P} \otimes \mathscr{F}^{q}\right)$ is locally expressible on $W \subset E$ as a sum of terms of the form

$$
\omega=\left(\sum_{|\alpha|=p} \omega_{1}^{\alpha} d x_{\alpha}\right) \otimes\left(\sum_{|\beta|=q} \omega_{1}^{\beta} d y_{\beta}^{v}\right)
$$


so that

$$
\Omega(\omega)=\sum_{\substack{\alpha|=p\\| \beta \mid=q}}\left(\omega_{1}^{\alpha} \Pi_{E}\right) \omega_{2}^{\beta} d x_{\alpha} \wedge d y_{\beta}^{v}
$$

Now we may write on $W$

$$
\begin{aligned}
& d y_{j}^{v}=d y_{j}+\sum_{i} \theta_{j}^{i} d x_{i} \\
& d y_{k}=d y_{k}^{v}+\sum_{j} \zeta_{k}^{j} d x_{j}
\end{aligned}
$$

where $\theta_{j}^{i}, \zeta_{k}^{j} \in C^{\infty}(W)$.

Replacing $d y_{\beta}^{v}$ using equation (7) one obtains

$$
\Omega(\omega)=\sum_{\substack{|\alpha=p\\| \beta \mid=q}}\left(\omega_{1}^{\alpha} \Pi_{E}\right) \omega_{2}^{\beta} d x_{\alpha} \wedge d y^{\beta}+\sum_{\substack{|\alpha|>p \\ \mu}}(\cdots)^{\alpha \mu} d x_{\alpha} \wedge d y_{\mu}
$$

(where $(\cdots)^{\alpha \mu}$ indicate coefficients of terms with $|\alpha|>p$ ) so that using the definition of $\rho^{p, q+1}$, one obtains on account of $\partial / \partial y_{k}\left(\omega_{1}^{\alpha} \Pi_{E}\right)=0$,

$$
\Delta_{0}^{p, q} \Omega(\omega)=\rho^{p, q+1} \delta \Omega(\omega)=\sum_{\substack{\alpha|=p\\| \beta \mid=q}} \sum_{k} \frac{\partial \omega_{2}^{\beta}}{\partial y_{k}}\left(\omega_{1}^{\alpha} \Pi_{E}\right) d y_{k}^{v} \wedge d x_{\alpha} \wedge d y_{\beta}^{v}
$$

One sees immediately that this is the same as the expression for $\Omega \delta_{F}^{p, q}(\omega)$, proving Lemma 3 .

As a consequence we have

$$
E_{1}^{p, q}=\operatorname{Ker} \Delta_{0}^{p, q} / \operatorname{Im} \Delta_{0}^{p, q-1}=\operatorname{Ker} \delta_{F}^{p, q} / \operatorname{Im} \delta_{F}^{p, q-1} .
$$

Let $h^{q}$ be the sheaf of germs of smooth sections of the bundle $h^{q}$. Now one can show by an argument identical to the one employed by Borel ([6], pp. 206, 207) that there is an isomorphism

$$
h^{q}=\operatorname{Ker} \delta_{F}^{q} / \operatorname{Im} \delta_{F}^{q-1},
$$

and hence also $\operatorname{Ker} \delta_{F}^{p, q} / \operatorname{Im} \delta_{E}^{p, q-1} \cong A_{B}^{p}\left(h^{q}\right)$. Thus we have

LEMMA 4. $E_{1}^{p, q} \cong A_{B}^{p}\left(h^{q}\right)$.

Proof of Proposition 2. Because $H^{p}\left(B ; h^{q}\right)=\operatorname{Ker} \delta_{B}^{p, q} / \operatorname{Im} \delta_{B}^{p-1, q}$ (see $\S 1$ (a) for the definition of $\left.\delta_{B}^{p, q}\right)$ and $E_{2}^{p, q} \cong \operatorname{Ker} \Delta_{1}^{p, q} / \operatorname{Im} \Delta_{1}^{p-1, q}$ it suffices to show that the diagram (11) below is commutative:

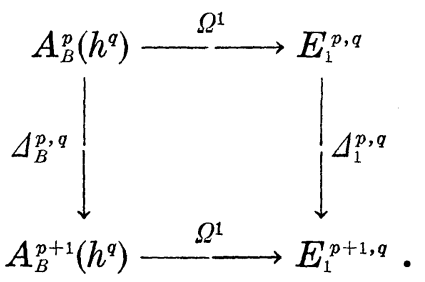


Here $\Omega^{1}$ is the isomorphism induced by virture of Lemma 4 . Consider the exact sequence of cochain complexes

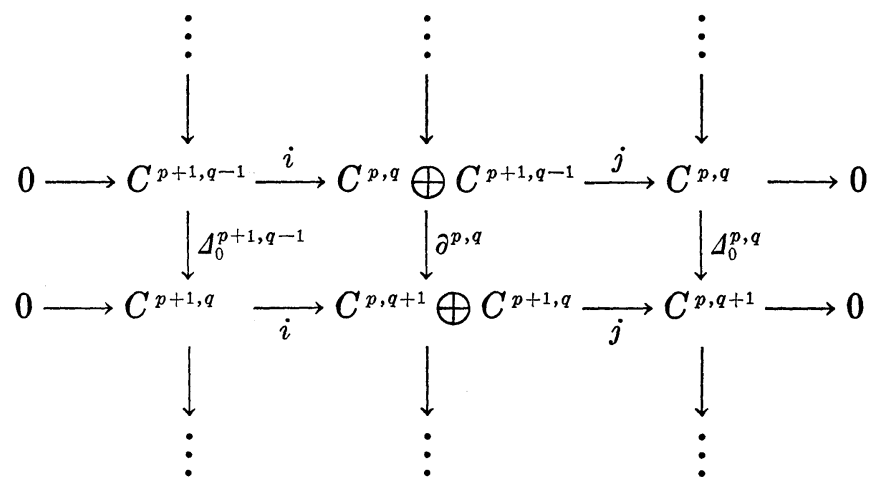

where $\partial^{p, q}$ is induced by the exterior derivative $\delta: A(E) \rightarrow A(E)$ and $i(d)=(0, d), d \in C^{p+1, q-1}, j(c, d)=c, c \in C^{p, q}$.

Now it is known $\left([3]\right.$, p. 85) that the differential operator $\Delta_{1}^{p, q}$ is the same as the connecting homomorphism $d^{p, q}$ induced by this exact sequence:

$$
d^{p, q}: \operatorname{Ker} \Delta_{0}^{p, q} / \operatorname{Im} \Delta_{0}^{p, q-1} \longrightarrow \operatorname{Ker} \Delta_{0}^{p+1, q} / \operatorname{Im} \Delta_{0}^{p+1, p-1} .
$$

Consequently, if $\omega \in \operatorname{Ker} \Delta_{0}^{p, q}$

$$
\Delta_{1}^{p, q}\left([\omega]_{E_{1}^{p, q}}=\left[i^{-1} \partial^{p, q} j^{-1} \omega\right]_{E_{1}^{p+1, q}},\right.
$$

where $[\omega]_{E_{1}^{p, q}}$ indicates the class of $\omega$ in $E_{1}^{p, q}$, and so on; that is, $\Delta_{1}^{p, q}\left([\omega]_{E_{1}^{p, q}}\right)$ is $\partial^{p, q} \omega$ modulo $\operatorname{Im} \Delta_{0}^{p+1, q-1}$.

Now suppose that $\omega \in A_{B}^{p}\left(h^{q}\right)$; then for a coordinate neighbourhood $U \subset B, \omega / U$ may be written as

$$
\omega / U(x)=\sum_{\substack{|\alpha|=p \\|\beta|=q}}\left[\omega^{\alpha \beta} d y_{\beta}^{v}\right]_{\left.H_{c}^{p} F_{x}\right)} d x_{\alpha}
$$

where $x \in U \subset B$ and $\omega^{\alpha \beta} \in C^{\infty}\left(\Pi_{E}^{-1}(U)\right)$.

A consideration of the isomorphism $\Omega^{1}$ yields

$$
\begin{aligned}
\Omega^{1}(\omega / U) & =\sum_{\substack{|\alpha|=p \\
|\beta|=q}}\left[\omega^{\alpha \beta} d x_{\alpha} \wedge d y_{\beta}^{v}\right]_{E_{1}^{p}, q} \\
& =\left[\sum_{\substack{|\alpha|=p \\
|\beta|=q}} \omega^{\alpha \beta} d x_{\alpha} \wedge d y_{\beta}+\sum_{\substack{|\alpha|>p \\
\mu}}(\cdots)^{\alpha \mu} d x_{\alpha} \wedge d y_{\mu}\right]_{E_{1}^{p, q}},
\end{aligned}
$$

where we have again replaced $d y_{\beta}^{v}$ by means of equation (7).

A short computation now shows, when all quotients have been taken, and using (8), that 


$$
\Delta_{1}^{p, q} \Omega^{1}(\omega / U)=\left[\sum_{\substack{|\alpha|=p \\ \beta \mid=q}} \sum_{k} \frac{\partial \omega^{\alpha \beta}}{\partial x_{k}} d x_{k} \wedge d x_{\alpha} \wedge d y_{\beta}^{v}\right]_{E_{1}^{p+1, q}} ;
$$

on the other hand,

$$
\begin{aligned}
\Omega^{1} \delta_{B}^{p, q}(\omega / U) & =\Omega^{1} \delta_{B}^{p, q}\left(\sum_{\substack{|\alpha|=p \\
|\beta|=q}}\left[\omega^{\alpha \beta} d y_{\beta}^{v}\right]_{H_{c}^{q}(F)} d x_{\alpha}\right) \\
& =\Omega^{1}\left(\sum_{\substack{|\alpha|=p \\
|\beta|=q}} \sum_{k}\left[\frac{\partial \omega^{\alpha \beta}}{\partial x_{k}} d y_{\beta}^{v}\right]_{H_{c}^{q}(F)} d x_{k} \wedge d x_{\alpha}\right)
\end{aligned}
$$

where $\left[\omega^{\alpha \beta} d y_{\beta}^{v}\right]_{H_{c}^{q}(F)}$ indicates the section of $h^{q}$ over $U$ defined by

$$
\left[\omega^{\alpha \beta} d y_{\beta}^{v}\right]_{H_{c}^{q}(F)}(x)=\left[\omega^{\alpha \beta} d y_{\beta}^{\nu}\right]_{H_{c}^{q}\left(F_{x}\right)}, x \in U
$$

(recall that $\left(h^{q}\right)_{x}=H_{c}^{q}\left(F_{x}\right)$ ).

The expression above clearly yields the right hand side of equation (12), as required.

2. Fiber integration, algebraic definition. As mentioned earlier, algebraic fiber integration is defined by using the definition of Borel and Hirzebruch [1] applied to the spectral sequence $\left\{E_{r}\right\}$ arising from the filtration $\left\{I^{p}\right\}$ of fiber-compact forms on the total space of $\xi$.

For convenience we recall some definitions from the theory of (decreasing) spectral sequences:

$$
\begin{aligned}
& Z_{s}^{p, q}=A_{F}^{p, q}(E) \cap\left\{a \in I^{p} \mid \delta a \in I^{p+s}\right\} \\
& D_{s}^{p, q}=A_{F}^{p+q}(E) \cap I^{p} \cap \delta I^{p-s} \\
& E_{s}^{p, q}=Z_{s}^{p, q} /\left(Z_{s-1}^{p+1, q-1} \oplus D_{s-1}^{p, q}\right)
\end{aligned}
$$

where $0 \leqq p, q, s \leqq \infty$.

Let $\Pi: Z_{\infty}^{0} \rightarrow H_{F}(E)$ be the canonical projection; then

$$
H^{p, q}=H_{F}^{p+q}(E) \cap \Pi\left(Z_{\infty}^{p}\right) \text { filter } H_{F}(E) \text {, and } E_{\infty}^{p, q}=H_{\infty}^{p, q} / H_{\infty}^{p+1, q-1} \text {. }
$$

Because $\operatorname{dim} F=m$ and $\operatorname{dim} B=n-m$, it follows that $E_{r}^{p, q}=0$ for $q>m, p \geqq 0, r \geqq 0$ and that $I^{p}=0$ for $p>n-m$.

LEMMA 5. (a) $E_{r}^{p, m} \subset E_{r-1}^{p, m}, r \geqq 3, p \geqq 0$.

(b) $E_{r}^{k-m, q}=E_{\infty}^{k-m, q}, r>\sup (n-k, k-m), k \geqq m, q \geqq 0$.

(c) $E_{\infty}^{k-m, m}=H_{F}^{k}(E) / H_{\infty}^{k-m+1, m-1}, k \geqq m$.

As a consequence of Lemma 5 we now have an injection

$$
h_{1}: E_{\infty}^{k-m, m}=E_{r_{0}}^{k-m, m} \subset \cdots \subset E_{2}^{k-m, m}
$$

where $r_{0}=\sup (n-k, k-m)+1$, and a projection $h_{2}: H_{F}^{k}(E) \rightarrow E_{\infty}^{k-m, m}$. Let $\chi: E_{2}^{k-m, m} \cong H^{k-m}\left(B ; h^{m}\right)$ be the isomorphism induced by $\Omega$ (Proposition 2). Then the definition of Borel, Hirzebruch yields $\chi h_{1} h_{2}$ : 
$H_{F}^{k}(E) \rightarrow H^{k-m}\left(B ; h^{m}\right)$. We now define $\sigma: H^{k-m}\left(B ; h^{m}\right) \rightarrow H^{k-m}(B)$, under the assumption that $\xi$ is orientable, in the following sense (see [4]):

Definition 6. $\xi$ is orientable if, and only if, there exists an $m$ form $\psi$ on $E$ such that for all $x \in B, i_{x}^{*} \psi$ is an orientation on $F_{x}$, the fiber over $x ; i_{x}: F_{x} \subset E$. If such a $\psi$ has been chosen, $\xi$ is called oriented.

Clearly $F$ is orientable when $\xi$ is.

Recall that $\hbar^{m}=\operatorname{Ker} \delta_{F}^{m} / \operatorname{Im} \delta_{F}^{m-1}=\mathscr{F}^{m} / \operatorname{Im} \delta_{F}^{m-1}$.

We first define a map of presheaves, $\mathscr{I}: \mathscr{F}^{m} \rightarrow \mathscr{C}_{B}^{\infty}$, where $\mathscr{C}_{B}^{\infty}$ denotes the presheaf of germs of $C^{\infty}$-functions on $B$ : Let $x \in U$, $\bar{U}$ open in $B$, and $\omega \in \mathscr{F}^{m}(U)$; then $(\mathscr{F}(U)(\omega))(x)=\int_{F_{x}} \omega(x)$. To show this is well defined, let $\left\{U_{j} / j \in J\right\}$ be a covering of $B$ by open sets such that $E$ is trivial over each $U_{j}$, with $\phi_{j}: U_{j} \times F \cong \Pi_{E}^{-1}\left(U_{j}\right), j \in J$. Define $\psi_{j, x}: F \rightarrow F_{x}$ by $\psi_{j, x}(f)=\phi_{j}(x, f), x \in U_{j}, f \in F$. Then

$$
\left(\mathscr{F}\left(U_{j}\right)(\omega)\right)(x)=\operatorname{deg} \psi_{j, x} \int_{F} \psi_{j, x}^{*}(\omega(x)) ;
$$

this shows that $\mathscr{I}(U)(\omega)$ is $C^{\infty}$ in $x$, because, since $\omega$ has fibercompact support, the forms $\psi_{j, x}^{*}(\omega(x))$ on $F$ have supports contained in a common compact set for $x$ in sufficiently small open sets in $B$.

Thus there is a sheaf homomorphism $\mathscr{J}: \mathscr{F}^{m} \rightarrow \mathscr{C}_{B}^{\infty}$; if $\omega \in \operatorname{Im} \underline{\delta}_{F}^{m-1}(U)$, then by Stokes' Theorem, $(\mathscr{J}(U)(\omega))(x)=0$, for all $x \in U$. Consequently, $\mathscr{J}$ induces a sheaf homomorphism, also denoted by $\mathscr{I}, \mathscr{F}: \mathscr{F}^{m} / \operatorname{Im} \delta_{F}^{m-1}=h^{m} \rightarrow \mathscr{C}_{B}^{\infty}$. Lastly,

$$
\sigma: H^{k-m}\left(B ; h^{m}\right) \longrightarrow H^{k-m}(B)
$$

is canonically induced by $\mathscr{J}$ on account of the commutative diagram below:

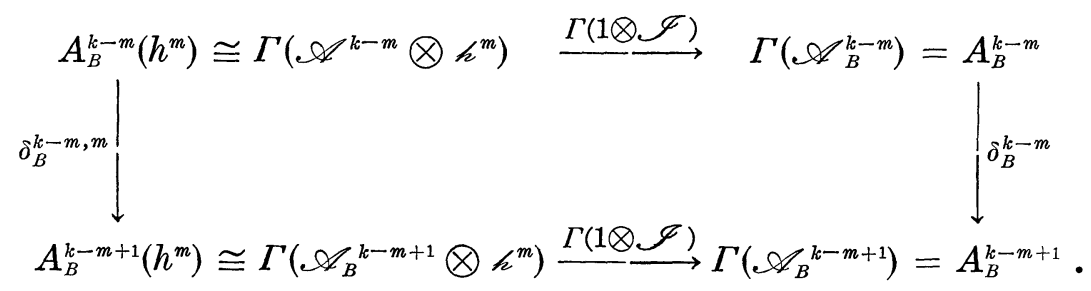

Combining $\sigma$ with the map $\chi h_{1} h_{2}$ we obtain algebraic fiber integration $\Psi_{1}=\sigma \chi h_{1} h_{2} ; \Psi_{1}: H_{F}^{k}(E) \rightarrow H^{k-m}(B)$.

3. Fiber integration, geometric definition ([4], chapter 7). For arbitrary manifolds $B, F$, and $x \in B, y \in F$, define 


$$
\begin{aligned}
& i_{x}: F \longrightarrow B \times F \text { by } i_{x}(y)=(x, y), y \in F, \\
& i_{y}: B \longrightarrow B \times F \text { by } i_{y}(x)=(x, y), x \in B .
\end{aligned}
$$

When $\xi \in T_{x}(B)$, put $\hat{\xi}=\left(i_{y}\right)_{*} \xi \in T_{(x, y)}(B \times F)$ and when $\zeta \in T_{y}(F)$, let $\hat{\zeta}=\left(i_{x}\right)_{*} \zeta \in T_{(x, y)}(B \times F)$.

Define $\lambda_{x}=C^{p, q}(B \times F) \rightarrow A_{c}^{q}\left(F ; \wedge^{p} T_{x}^{*}(B)\right)$ (with the trivial connection in the product bundle $B \times F$ ) by

$$
\left.\left(\lambda_{x} \omega\right)\left(y ; \zeta_{1}, \cdots, \zeta_{q}\right)\left(\xi_{1}, \cdots, \xi_{p}\right)=\omega\left((x, y) ; \hat{\xi}_{1}, \cdots, \hat{\xi}_{p}, \hat{\zeta}_{1}, \cdots, \hat{\zeta}_{q}\right)\right)
$$

where $x \in B, \omega \in C^{p, q}(B \times F), \zeta_{i} \in T_{y}(F)$ and $\xi_{j} \in T_{x}(B)$. For the product bundle $B \times F$ geometric fiber integration is the linear map

$$
f_{F}: C^{p, r}(B \times F) \longrightarrow A^{p}(B), p \geqq 0,
$$

defined by $\left(f_{F} \omega\right)(x)=\left\{\begin{array}{l}\int_{F} \lambda_{x} \omega, x \in B, r=m \\ 0, r \neq m,\end{array} \quad\right.$ where $\omega \in C^{p, r}(B \times F)$.

For an arbitrary oriented bundle $\xi$, let $\left\{U_{j}, \phi_{j}\right\}$ be a family of trivializations as before with $\phi_{j}: U_{j} \times F \cong \Pi_{E}^{-1}\left(U_{j}\right)$. If $\omega \in A_{F}^{k}(E)$, $\phi_{j}^{*} \omega$ is a fiber-compact form on $U_{j} \times F$, so that $\omega_{j}$ defined by $\omega_{j}(x)=$ $\operatorname{deg} \psi_{j, x}\left(f_{F} \phi_{j}^{*} \omega\right)(x)$ is a $k-m$ form on $U_{j}$. Define $f_{F} A_{F}^{k}(E) \rightarrow A^{k-m}(B)$ by $\left(f_{F^{\prime}}(\omega)\right)(x)=\omega_{j}(x)$ when $x \in U_{j}$.

It is easily shown that this is independent of the choice of $U_{j}$, so that $f_{F}$ is well defined ([4]).

Furthermore, $f_{F} \delta_{E}=\delta_{B} f_{E}$, so that there is an induced map

$$
\Psi_{2}: H_{F}^{k}(E) \longrightarrow H^{k-m}(B),
$$

$k \geqq m$, called geometric fiber integration.

4. Proof of Theorem. $\Psi_{1}=\Psi_{2}$.

Let $[\omega] \in H_{F}^{k}(E)$ be represented by $\omega \in A_{F}^{k}(E)$.

If $W_{j}=\Pi_{E}^{-1}\left(U_{j}\right)$ is sufficiently small we may write

$$
\omega / W_{j}=\sum_{|\alpha|+|\beta|=k} \omega^{\alpha \beta} d x_{\alpha} \wedge d y_{\beta}, \quad \omega^{\alpha \beta} \in C^{\infty}\left(W_{j}\right),
$$

or, upon substitution of equation (8) for $d y_{\beta}$,

$$
\omega / W_{j}=\sum_{\substack{|\alpha|=k-m \\|\beta|=m}} \omega^{\alpha \beta} d x_{\alpha} \wedge d y_{\beta}^{v}+\sum_{\substack{|\alpha|>k \rightarrow m \\ \beta}}(\cdots)^{\alpha \beta} d x_{\alpha} \wedge d y_{\beta}^{v} .
$$

Since $h_{2}: H_{F}^{k}(E) \rightarrow H_{F}^{k}(E) / H_{\infty}^{k-m+1, m-1}=E_{\infty}^{k-m, m}$ is merely the projection, 


$$
h_{2}([\omega])=\left[\sum_{\substack{\alpha|=k-m\\| \beta \mid=m}} \omega^{\alpha \beta} d x_{\alpha} \wedge d y_{\beta}^{v}\right]_{E_{\infty}^{k-m, m}}
$$

Hence,

$$
\chi h_{1} h_{2}([\omega])=\left[\sum_{\substack{|\alpha|=k-m \\|\beta|=m}}\left[\omega^{\alpha \beta} d y_{\beta}^{v}\right]_{h^{m}} d x_{\alpha}\right]_{H^{k-m}\left(B ; h^{m}\right)}
$$

and

$$
\Psi_{1}([\omega])=\sigma \chi h_{1} h_{2}([\omega])=[\mu]_{H^{k-m}(B)},
$$

where $\mu \in A^{k-m}(B)$ with

$$
\mu(x)=\sum_{|\alpha|=k-m}\left(\operatorname{deg} \psi_{j, x} \int \psi_{j, x}^{*}\left(\sum_{|\beta|=m} \omega^{\alpha \beta} d y_{\beta}^{v}\right)\right) d x^{\alpha}
$$

when $x \in U_{j}$.

On the other hand,

$$
\Psi_{2}([\omega])=\left[f_{F} \omega\right]_{H^{k-m}(B)}
$$

and

$$
\left(f_{F} \omega\right)(x)=\operatorname{deg} \psi_{j, x} \int_{F} \lambda_{x}\left(\phi_{j}^{*} \omega\right)=\operatorname{deg} \psi_{j, x} \int_{F}\left(\sum_{\substack{\alpha|=k-m\\| \beta \mid=m}} \omega^{\alpha \beta} \psi_{j, x} d y_{\beta}\right) d x_{\alpha}
$$

as a short computation shows.

A comparison of (16), (17) shows that $\Psi_{1}=\Psi_{2}$ as required.

\section{REFERENCES}

1. A. Borel and F. Hirzebruch, Characteristic classes and homogeneous spaces $I$, Amer. J. Math. 80 (1958), 458-538.

2. S. S. Chern, On the characteristic classes of complex sphere bundles and algebraic varieties, Amer. J. Math., 75 (1953), 565-597.

3. R. Godement, Topologie Algébrique et Théorie des Faisceaux, Hermann (1964).

4. W. H. Grueb, S. Halperin, and R. Vanstone, Cohomology of Smooth Bundles I, II, III, Academic Press (to appear).

5. A. Hattori, A Spectral sequence in the de Rham cohomology of fibre bundles, J. Fac. Sci. Univ. Tokyo Sect. I (1960), 289-331.

6. F. Hirzebruch, Topological Methods in Algebraic Geometry, Springer Verlag (1966) appendix II.

7. S. Kobayashi and K. Nomizu, Foundations of Differential Geometry, volume I, Interscience (1963).

Received September 30, 1971.

BROCK UNIVERSITY 



\section{PACIFIC JOURNAL OF MATHEMATICS}

\section{EDITORS}

H. SAMELSON

Stanford University

Stanford, California 94305

C. R. Hовву

University of Washington

Seattle, Washington 98105
J. DugundJI

Department of Mathematics University of Southern California

Los Angeles, California 90007

RICHARD ARENS

University of California

Los Angeles, California 90024

\section{ASSOCIATE EDITORS}

E. F. BECKENBACH

B. H. NeumanN

F. WOLF

K. YoSHIDA

\section{SUPPORTING INSTITUTIONS}

UNIVERSITY OF BRITISH COLUMBIA

CALIFORNIA INSTITUTE OF TECHNOLOGY

UNIVERSITY OF CALIFORNIA

MONTANA STATE UNIVERSITY

UNIVERSITY OF NEVADA

NEW MEXICO STATE UNIVERSITY

OREGON STATE UNIVERSITY

UNIVERSITY OF OREGON

OSAKA UNIVERSITY
UNIVERSITY OF SOUTHERN CALIFORNIA

STANFORD UNIVERSITY

UNIVERSITY OF TOKYO

UNIVERSITY OF UTAH

WASHINGTON STATE UNIVERSITY

UNIVERSITY OF WASHINGTON

AMERICAN MATHEMATICAL SOCIETY

NAVAL WEAPONS CENTER

Printed in Japan by International Academic Printing Co., Ltd., Tokyo, Japan 


\section{Pacific Journal of Mathematics}

\section{Vol. 44, No. $1 \quad$ May, 1973}

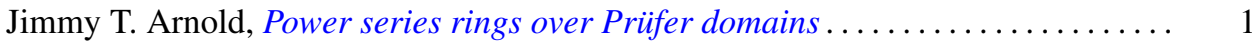

Maynard G. Arsove, On the behavior of Pincherle basis functions . . . . . . . . . 13

Jan William Auer, Fiber integration in smooth bundles ................. 33

George Bachman, Edward Beckenstein and Lawrence Narici, Function algebras

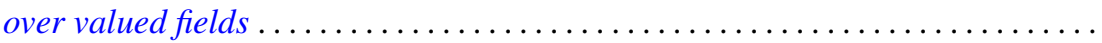

Gerald A. Beer, The index of convexity and the visibility function . . . . . . . . . . .

James Robert Boone, A note on mesocompact and sequentially mesocompact

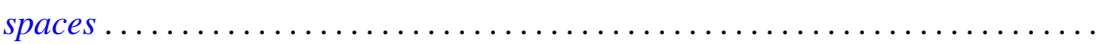

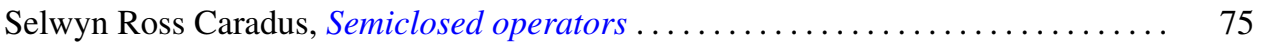

John H. E. Cohn, Two primary factor inequalities . . . . . . . . . . . . . . . 81

Mani Gagrat and Somashekhar Amrith Naimpally, Proximity approach to

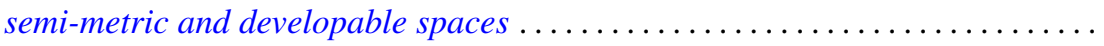

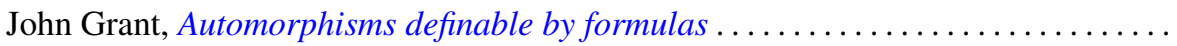

Walter Kurt Hayman, Differential inequalities and local valency ..............

Wolfgang H. Heil, Testing 3-manifolds for projective planes . . . . . . . . . . . . .

107

Melvin Hochster and Louis Jackson Ratliff, Jr., Five theorems on Macaulay

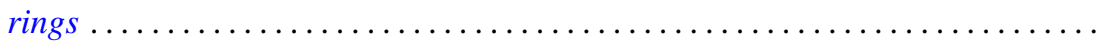

Thomas Benton Hoover, Operator algebras with reducing invariant subspaces ....

James Edgar Keesling, Topological groups whose underlying spaces are separable

Fréchet manifolds...

Frank Leroy Knowles, Idempotents in the boundary of a Lie group . .

191

George Edward Lang, The evaluation map and EHP sequences ...

201

Everette Lee May, Jr, Localizing the spectrum . . . . . . . . . . . .

211

Frank Belsley Miles, Existence of special $K$-sets in certain locally compact abelian groups.

Susan Montgomery, A generalization of a theorem of Jacobson. II . .

T. S. Motzkin and J. L. Walsh, Equilibrium of inverse-distance forces in

three-dimensions.

Arunava Mukherjea and Nicolas A. Tserpes, Invariant measures and the converse

of Haar's theorem on semitopological semigroups .

James Waring Noonan, On close-to-convex functions of order $\beta$

Donald Steven Passman, The Jacobian of a growth transformation

Dean Blackburn Priest, A mean Stieltjes type integral ........ .

Joe Bill Rhodes, Decomposition of semilattices with applications to topological

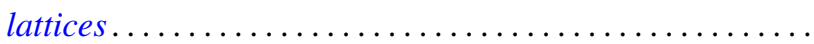

Claus M. Ringel, Socle conditions for $\mathrm{QF}-1$ rings ..........

Richard Rochberg, Linear maps of the disk algebra

Roy W. Ryden, Groups of arithmetic functions under Dirichlet convolution . .

Michael J. Sharpe, A class of operators on excessive functions

Erling Stormer, Automorphisms and equivalence in von Neumann algebras ..

Philip C. Tonne, Matrix representations for linear transformations on series

analytic in the unit disc. 